Research Article

\title{
Determination of Number of Infected Cells and Concentration of Viral Particles in Plasma during HIV-1 Infections Using Shehu Transformation
}

\author{
M. Higazy, ${ }^{1,2}$ Sudhanshu Aggarwal $\mathbb{D}^{3},{ }^{3}$ and Y. S. Hamed $\mathbb{D}^{1,2}$ \\ ${ }^{1}$ Department of Mathematics and Statistics, College of Science, Taif University, P.O. Box 11099, Taif 21944, Saudi Arabia \\ ${ }^{2}$ Department of Physics and Engineering Mathematics, Faculty of Electronic Engineering, Menoufia University, \\ Menouf 32952, Egypt \\ ${ }^{3}$ Department of Mathematics, National P.G. College, Barhalganj, Gorakhpur 273402, Uttra Pradesh, India
}

Correspondence should be addressed to Sudhanshu Aggarwal; sudhanshu30187@gmail.com

Received 10 October 2020; Revised 4 November 2020; Accepted 24 November 2020; Published 7 December 2020

Academic Editor: Hijaz Ahmad

Copyright (c) 2020 M. Higazy et al. This is an open access article distributed under the Creative Commons Attribution License, which permits unrestricted use, distribution, and reproduction in any medium, provided the original work is properly cited.

In this paper, the authors determine the number of infected cells and concentration of infected (viral) particles in plasma during HIV-1 (human immunodeficiency virus type one) infections using Shehu transformation. For this, the authors first defined some useful properties of Shehu transformation with proof and then applied Shehu transformation on the mathematical representation of the HIV-1 infection model. The mathematical model of HIV-1 infections contains a system of two simultaneous ordinary linear differential equations with initial conditions. Results depict that Shehu transformation is very effective integral transformation for determining the number of infected cells and concentration of viral particles in plasma during HIV-1 infections.

\section{Introduction}

Phylogenetically, two types of HIV are recognized, HIV-1 and HIV-2. There is evidence [1-3] which supports the hypothesis that HIV-1 was first transmitted from chimpanzees to man and HIV-2 transferred from sooty mangabey monkey to humans. Today, there are millions of people infected by HIV and many more each day have their lives affected by it.

Researchers [1] have now concluded that HIV is not an airborne virus like that influenza virus. HIV is not transmitted by insects such as fleas, and it is not transmitted by mosquitoes. HIV is not transmitted by hugging, touching, and shaking hands with infected persons. HIV is transmitted in humans through four main body fluids: semen, blood, breast milk, and vaginal secretions. Other unusual case of HIV transmission has been documented, but upon investigation, all have involved some exchange of one of these infected body fluids.

$\mathrm{HIV}$ is a virus. Viruses are microscopic biological agents that are technically not considered to be living, as they do not meet the scientific requirements for what constitutes life. They are only able to replicate and reproduce themselves through the use of other cells. Specially, they infect themselves to a host cell and deliver their genetic material to the cell. They then hijack the cells' mechanisms for reproduction and use the cell to replicate many versions of themselves. So, it is very essential to determine the number of infected cells and concentration of viral particles in plasma during HIV-1 infections.

Integral transformation is one of the linchpins of modern science and engineering which is essential for finding the exact solutions of complex problems which appears in engineering, business, natural sciences, optical science, computers, and modern mathematics. Nowadays, Integral transformations play a predominant role in mathematics, medical science, chemical engineering, radar and signal processing, physics, fluid mechanics, and theory of elasticity $[4,5]$.

Many scholars [6, 7] used different integral transformations for obtaining the solutions of complex problems of 
mathematics, biology, chemistry, mechanics, physics, etc. Recently, Aggarwal with others [8-10] comparatively discussed many new integral transformations and determined the solutions of system of simultaneous ordinary linear differential equations by applying these new integral transformations on them. Maitama and Zhao [11] solved constant differential equations by defining Shehu transformation. In year 2019, Aggarwal and others [12-14] used Shehu transformation and determined the solutions of many problems such as growth and decay problems, famous Abel's problem of mechanics, and linear first kind Volterra integral equations. Aggarwal and Singh [15] defined Shehu transformation of probability integral which frequently appears in statistics. Many complex problems of medicine and electrical engineering such as problem of determining total red blood cells in a hospital patient undergoing surgery and problem of two-loop electrical circuit can be modeled using differential equation and their system $[16,17]$. Perelson et al. [18] discussed the HIV-1 model with the help of system of differential equations. Mathematical analysis of HIV-1 dynamics in vivo was given by Perelson and Nelson [19]. Perelson [20] gave the modeling of viral and immune system. Nelson and Perelson [21] used delay differential equation and analyzed the HIV-1 infection model. Stafford et al. [22] discussed the plasma virus concentration during primary HIV infection. Rong et al. [23] gave the mathematical modeling of HIV-1 infection and drug therapy. Aggarwal et al. [24] applied Laplace transform for determining the solutions of population growth and decay problems.

To determine the number of infected cells and concentration of viral particles in plasma during HIV-1 infections by applying Shehu transformation on the mathematical model of HIV-1 infections is the main aim of this paper.

\section{Definition of Shehu Transformation}

The Shehu transformation of the function $\omega(t), t \geq 0$ is given by [11]

$$
S\{\omega(t)\}=\int_{0}^{\infty} \omega(t) e^{-(\varepsilon / \sigma) t} \mathrm{~d} t=T(\varepsilon, \sigma), \quad \varepsilon>0, \sigma>0 .
$$

\section{Some Important Properties of Shehu Transform}

The authors present important characteristics of Shehu transformation in this part of the paper.

3.1. Linearity Property of Shehu Transformation. If Shehu transform of functions $\omega_{1}(t)$ and $\omega_{2}(t)$ is $T_{1}(\varepsilon, \sigma)$ and $T_{2}(\varepsilon, \sigma)$, respectively, then Shehu transform of $\left[l \omega_{1}(t)+\right.$ $\left.m \omega_{2}(t)\right]$ is given by $\left[l T_{1}(\varepsilon, \sigma)+m T_{2}(\varepsilon, \sigma)\right]$, where $l, m$ are arbitrary constants.

Proof. Using (1), we obtain

$$
\begin{aligned}
& S\{\omega(t)\}=\int_{0}^{\infty} \omega(t) e^{-(\varepsilon / \sigma) t} \mathrm{~d} t, \\
& \Longrightarrow S\left\{l \omega_{1}(t)+m \omega_{2}(t)\right\}=\int_{0}^{\infty}\left[l \omega_{1}(t)\right. \\
& \left.+m \omega_{2}(t)\right] e^{-(\varepsilon / \sigma) t} \mathrm{~d} t \\
& \Longrightarrow S\left\{l \omega_{1}(t)+m \omega_{2}(t)\right\}=l \int_{0}^{\infty} \omega_{1}(t) e^{-(\varepsilon / \sigma) t} \mathrm{~d} t \\
& +m \int_{0}^{\infty} \omega_{2}(t) e^{-(\varepsilon / \sigma) t} \mathrm{~d} t \\
& \Longrightarrow S\left\{l \omega_{1}(t)+m \omega_{2}(t)\right\}=l S\left\{\omega_{1}(t)\right\}+m S\left\{\omega_{2}(t)\right\} \\
& \Longrightarrow S\left\{l \omega_{1}(t)+m \omega_{2}(t)\right\}=l T_{1}(\varepsilon, \sigma)+m T_{2}(\varepsilon, \sigma) \text {, }
\end{aligned}
$$

where $l, m$ are arbitrary constants.

3.2. Scaling Property of Shehu Transformation. If $S\{\omega(t)\}=T(\varepsilon, \sigma)$, then $S\{\omega(k t)\}=(1 / k) T((\varepsilon / k), \sigma)$.

Proof. Using (1), we obtain

$$
\begin{aligned}
S\{\omega(t)\} & =\int_{0}^{\infty} \omega(t) e^{-(\varepsilon / \sigma) t} \mathrm{~d} t \\
& \Longrightarrow S\{\omega(k t)\}=\int_{0}^{\infty} \omega(k t) e^{-(\varepsilon / \sigma) t} \mathrm{~d} t .
\end{aligned}
$$

Substituting $k t=p \Longrightarrow k d t=d p$ in (3), we have

$$
\begin{aligned}
S\{\omega(k t)\} & =\frac{1}{k} \int_{0}^{\infty} \omega(p) e^{-(\varepsilon / \sigma)(p / k)} \mathrm{d} p \\
& \Longrightarrow S\{\omega(k t)\}=\frac{1}{k} \int_{0}^{\infty} e^{-(\varepsilon / k)(p / \sigma)} \omega(p) \mathrm{d} p \\
& \Longrightarrow S\{\omega(k t)\}=\frac{1}{k} T\left(\frac{\varepsilon}{k}, \sigma\right) .
\end{aligned}
$$

3.3. Translation Property of Shehu Transformation. If $S\{\omega(t)\}=T(\varepsilon, \sigma)$, then $S\left\{e^{k t} \omega(t)\right\}=T(\varepsilon-k \sigma, \sigma)$.

Proof. Using (1), we obtain

$$
\begin{aligned}
S\{\omega(t)\} & =\int_{0}^{\infty} \omega(t) e^{-(\varepsilon / \sigma) t} \mathrm{~d} t \\
& \Longrightarrow S\left\{e^{k t} \omega(t)\right\}=\int_{0}^{\infty} e^{k t} \omega(t) e^{-(\varepsilon / \sigma) t} \mathrm{~d} t \\
& \Longrightarrow S\left\{e^{k t} \omega(t)\right\}=\int_{0}^{\infty} \omega(t) e^{-(\varepsilon / \sigma-k) t} \mathrm{~d} t \\
& =\int_{0}^{\infty} \omega(t) e^{-(\varepsilon-k \sigma / \sigma) t} \mathrm{~d} t=T(\varepsilon-k \sigma, \sigma) .
\end{aligned}
$$

\section{Shehu Transformation of Derivatives}

If $S\{\omega(t)\}=T(\varepsilon, \sigma)$, then

(a) $S\left\{\omega^{\prime}(t)\right\}=(\varepsilon / \sigma) T(\varepsilon, \sigma)-\omega(0)$

(b) $S\left\{\omega^{\prime \prime}(t)\right\}=\left(\varepsilon^{2} / \sigma^{2}\right) T(\varepsilon, \sigma)-(\varepsilon / \sigma) \omega(0)-\omega^{\prime}(0)$ 

(c) $S\left\{\omega^{(\rho)}(t)\right\}=\left(\varepsilon^{\rho} / \sigma^{\rho}\right) T(\varepsilon, \sigma)-\sum_{k=0}^{\rho-1}(\varepsilon / \sigma)^{\rho-(k+1)} \omega^{(k)}$
(b) We have

Proof

(a) Using (1), we obtain

$$
\begin{aligned}
S\{\omega(t)\}= & \int_{0}^{\infty} \omega(t) e^{-(\varepsilon / \sigma) t} \mathrm{~d} t \\
\Longrightarrow & S\left\{\omega^{\prime}(t)\right\}=\int_{0}^{\infty} \omega^{\prime}(t) e^{-(\varepsilon / \sigma) t} \mathrm{~d} t=\left[\omega(t) e^{-(\varepsilon / \sigma) t}\right]_{0}^{\infty} \\
& -\int_{0}^{\infty}\left\{-\left(\frac{\varepsilon}{\sigma}\right)\right\} \omega(t) e^{-(\varepsilon / \sigma) t} \mathrm{~d} t \\
= & \lim _{t \longrightarrow \infty}\left[\omega(t) e^{-(\varepsilon / \sigma) t}\right]-\omega(0)+\frac{\varepsilon}{\sigma} \int_{0}^{\infty} \omega(t) e^{-(\varepsilon / \sigma) t} \mathrm{~d} t \\
= & 0-\omega(0)+\frac{\varepsilon}{\sigma} S\{\omega(t)\} \\
\Longrightarrow & S\left\{\omega^{\prime}(t)\right\}=\frac{\varepsilon}{\sigma} T(\varepsilon, \sigma)-\omega(0) .
\end{aligned}
$$

$$
\begin{aligned}
S\left\{\omega^{\prime}(t)\right\} & =\frac{\varepsilon}{\sigma} T(\varepsilon, \sigma)-\omega(0)=\frac{\varepsilon}{\sigma} S\{\omega(t)\}-\omega(0) \\
& \Longrightarrow S\left\{\omega^{\prime \prime}(t)\right\}=\frac{\varepsilon}{\sigma} S\left\{\omega^{\prime}(t)\right\}-\omega^{\prime}(0) \\
& =\frac{\varepsilon}{\sigma}\left[\frac{\varepsilon}{\sigma} S\{\omega(t)\}-\omega(0)\right]-\omega^{\prime}(0) \\
& \Longrightarrow S\left\{\omega^{\prime \prime}(t)\right\}=\frac{\varepsilon^{2}}{\sigma^{2}} S\{\omega(t)\}-\frac{\varepsilon}{\sigma} \omega(0)-\omega^{\prime}(0) \\
& \Longrightarrow S\left\{\omega^{\prime \prime}(t)\right\}=\frac{\varepsilon^{2}}{\sigma^{2}} T(\varepsilon, \sigma)-\frac{\varepsilon}{\sigma} \omega(0)-\omega^{\prime}(0) .
\end{aligned}
$$

(c) We have

$$
\begin{aligned}
S\left\{\omega^{\prime \prime}(t)\right\} & =\frac{\varepsilon^{2}}{\sigma^{2}} T(\varepsilon, \sigma)-\frac{\varepsilon}{\sigma} \omega(0)-\omega^{\prime}(0)=\frac{\varepsilon^{2}}{\sigma^{2}} S\{\omega(t)\}-\frac{\varepsilon}{\sigma} \omega(0)-\omega^{\prime}(0) \\
& \Longrightarrow S\left\{\omega^{\prime \prime \prime}(t)\right\}=\frac{\varepsilon^{2}}{\sigma^{2}} S\left\{\omega^{\prime}(t)\right\}-\frac{\varepsilon}{\sigma} \omega^{\prime}(0)-\omega^{\prime \prime}(0)=\frac{\varepsilon^{2}}{\sigma^{2}}\left[\frac{\varepsilon}{\sigma} S\{\omega(t)\}-\omega(0)\right]-\frac{\varepsilon}{\sigma} \omega^{\prime}(0)-\omega^{\prime \prime}(0) \\
& \Longrightarrow S\left\{\omega^{\prime \prime \prime}(t)\right\}=\frac{\varepsilon^{3}}{\sigma^{3}} S\{\omega(t)\}-\frac{\varepsilon^{2}}{\sigma^{2}} \omega(0)-\frac{\varepsilon}{\sigma} \omega^{\prime}(0)-\omega^{\prime \prime}(0) \\
& =\frac{\varepsilon^{3}}{\sigma^{3}} T(\varepsilon, \sigma)-\frac{\varepsilon^{2}}{\sigma^{2}} \omega(0)-\frac{\varepsilon}{\sigma} \omega^{\prime}(0)-\omega^{\prime \prime}(0) .
\end{aligned}
$$

In general, we have

$$
\begin{aligned}
S\left\{\omega^{(\rho)}(t)\right\} & =\frac{\varepsilon^{\rho}}{\sigma^{\rho}} S\{\omega(t)\}-\frac{\varepsilon^{\rho-1}}{\sigma^{\rho-1}} \omega(0)-\frac{\varepsilon^{\rho-2}}{\sigma^{\rho-2}} \omega^{\prime}(0)-\cdots \cdots \cdots-\omega^{(\rho-1)}(0) \\
& \Longrightarrow S\left\{\omega^{(\rho)}(t)\right\}=\frac{\varepsilon^{\rho}}{\sigma^{\rho}} T(\varepsilon, \sigma)-\frac{\varepsilon^{\rho-1}}{\sigma^{\rho-1}} \omega(0)-\frac{\varepsilon^{\rho-2}}{\sigma^{\rho-2}} \omega^{\prime}(0)-\cdots \cdots \cdots-\omega^{(\rho-1)}(0) \\
& \Longrightarrow S\left\{\omega^{(\rho)}(t)\right\}=\frac{\varepsilon^{\rho}}{\sigma^{\rho}} T(\varepsilon, \sigma)-\sum_{k=0}^{\rho-1}\left(\frac{\varepsilon}{\sigma}\right)^{\rho-(k+1)} \omega^{(k)}(0) .
\end{aligned}
$$




\section{Inverse Shehu Transformation}

The inverse Shehu transformation of $T(\varepsilon, \sigma)$ designated by $S^{-1}\{T(\varepsilon, \sigma)\}$ is another function $\omega(t)$ having the property that $S\{\omega(t)\}=T(\varepsilon, \sigma)$.

\section{Linearity Property of Inverse Shehu Transformation}

If $S^{-1}\left\{T_{1}(\varepsilon, \sigma)\right\}=\omega_{1}(t)$ and $S^{-1}\left\{T_{2}(\varepsilon, \sigma)\right\}=\omega_{2}(t)$, then

$$
\begin{aligned}
S^{-1}\left\{l T_{1}(\varepsilon, \sigma)+m T_{2}(\varepsilon, \sigma)\right\} & =l S^{-1}\left\{T_{1}(\varepsilon, \sigma)\right\}+m S^{-1}\left\{T_{2}(\varepsilon, \sigma)\right\} \\
& \Longrightarrow S^{-1}\left\{l T_{1}(\varepsilon, \sigma)+m T_{2}(\varepsilon, \sigma)\right\}=l \omega_{1}(t)+m \omega_{2}(t)
\end{aligned}
$$

where $l, m$ are arbitrary constants.

\section{Relationship between Shehu and Laplace Transformations}

If $S\{\omega(t)\}=T(\varepsilon, \sigma)$ and $L\{\omega(t)\}=U(\varepsilon)$ where $L\{\omega(t)\}=$ $\int_{0}^{\infty} e^{-\varepsilon t} \omega(t) \mathrm{d} t=U(\varepsilon)$ is the Laplace transformation of $\omega(t)$, then $T(\varepsilon, \sigma)=U(\varepsilon / \sigma)$.

\section{Determination of the Total Number of Infected Cells and Concentration of Viral Particles in Plasma during HIV-1 Infections Using Shehu Transformation}

The authors present Shehu transformation for determining the number of infected cells and concentration of viral particles in plasma during HIV-1 infections in this part of the paper.

Perelson et al. [18] gave the mathematical model of HIV1 infections as

$$
\left.\begin{array}{l}
\frac{d \omega}{\mathrm{d} t}=\alpha \varphi \omega_{0}-\beta \omega \\
\frac{d \varphi}{\mathrm{d} t}=-\gamma \varphi
\end{array}\right\}
$$

with

$$
\begin{aligned}
& \omega(0)=\omega_{0}, \\
& \varphi(0)=\varphi_{0},
\end{aligned}
$$

where

$\omega=\omega(t)=$ number of infected cells at any time $\omega_{0}=$ number of potentially infected cells at the begining of therapy $\varphi=\varphi(t)=$ concentration of viral particles in plasma $\varphi_{0}=$ initial concentration of viral particles in plasma $\alpha=$ rate of infections

$\beta=$ rate of loss of virus producing cells

$\gamma=$ rate constant of viral particle clearance

The matrix representation of the above system is

$$
\frac{d L}{\mathrm{~d} t}=\mathrm{ML}+N(t) \text { with } L(0)=R,
$$

Where $(d L / \mathrm{d} t)=\left[\begin{array}{c}(d \omega / \mathrm{d} t) \\ (d \varphi / \mathrm{d} t)\end{array}\right], M=\left[\begin{array}{cc}-\beta & \alpha \omega_{0} \\ 0 & -\gamma\end{array}\right], L=\left[\begin{array}{l}\omega \\ \varphi\end{array}\right]$, $N=\left[\begin{array}{l}0 \\ 0\end{array}\right], L(0)=\left[\begin{array}{c}\omega(0) \\ \varphi(0)\end{array}\right]$, and $R=\left[\begin{array}{l}\omega_{0} \\ \varphi_{0}\end{array}\right]$.

Taking Shehu transformation of system (11) and using initial condition (12), we have

$$
\left.\begin{array}{l}
\left(\frac{\varepsilon}{\sigma}+\beta\right) S\{\omega\}-\alpha \omega_{0} S\{\varphi\}=\omega_{0} \\
\left(\frac{\varepsilon}{\sigma}+\gamma\right) S\{\varphi\}=\varphi_{0}
\end{array}\right\} .
$$

The primitives of (14) are determined by 


$$
\begin{aligned}
S\{\omega\} & =\frac{\left|\begin{array}{cc}
\omega_{0} & -\alpha \omega_{0} \\
\varphi_{0} & ((\varepsilon / \sigma)+\gamma)
\end{array}\right|}{\left|\begin{array}{cc}
((\varepsilon / \sigma)+\beta) & -\alpha \omega_{0} \\
0 & ((\varepsilon / \sigma)+\gamma)
\end{array}\right|}=\frac{\omega_{0} \sigma\left[(\varepsilon+\sigma \gamma)+\varphi_{0} \alpha \sigma\right]}{(\varepsilon+\sigma \gamma)(\varepsilon+\beta \sigma)}=\left[\frac{\omega_{0} \sigma}{(\varepsilon+\beta \sigma)}+\frac{\omega_{0} \varphi_{0} \alpha \sigma^{2}}{(\varepsilon+\sigma \gamma)(\varepsilon+\beta \sigma)}\right] \\
& =\left[\frac{\omega_{0} \sigma}{\varepsilon+\beta \sigma}+\frac{\omega_{0} \varphi_{0} \alpha}{(\beta-\gamma)}\left\{\frac{\sigma}{\varepsilon+\gamma \sigma}-\frac{\sigma}{\varepsilon+\beta \sigma}\right\}\right], \\
S\{\varphi\} & =\frac{\left|\begin{array}{cc}
((\varepsilon / \sigma)+\beta) & \omega_{0} \\
0 & \varphi_{0}
\end{array}\right|}{\left|\begin{array}{cc}
((\varepsilon / \sigma)+\beta) & -\alpha \omega_{0} \\
0 & ((\varepsilon / \sigma)+\gamma)
\end{array}\right|}=\frac{\varphi_{0}(1+\beta \sigma)}{\sigma(1+\sigma \gamma)(1+\beta \sigma)}=\frac{\varphi_{0} \sigma}{\varepsilon+\gamma \sigma} .
\end{aligned}
$$

Taking inverse Shehu transformation of equations (15) and (16), we have

$$
\begin{aligned}
\omega= & S^{-1}\left\{\left[\frac{\omega_{0} \sigma}{\varepsilon+\beta \sigma}+\frac{\omega_{0} \varphi_{0} \alpha}{(\beta-\gamma)}\left\{\frac{\sigma}{\varepsilon+\gamma \sigma}-\frac{\sigma}{\varepsilon+\beta \sigma}\right\}\right]\right\} \\
= & \omega_{0} S^{-1}\left\{\frac{\sigma}{\varepsilon+\beta \sigma}\right\}+\frac{\omega_{0} \varphi_{0} \alpha}{(\beta-\gamma)} S^{-1}\left\{\frac{\sigma}{\varepsilon+\gamma \sigma}-\frac{\sigma}{\varepsilon+\beta \sigma}\right\} \\
= & \omega_{0} S^{-1}\left\{\frac{\sigma}{\varepsilon+\beta \sigma}\right\}+\frac{\omega_{0} \varphi_{0} \alpha}{(\beta-\gamma)}\left[S^{-1}\left\{\frac{\sigma}{\varepsilon+\gamma \sigma}\right\}\right. \\
& \left.-S^{-1}\left\{\frac{\sigma}{\varepsilon+\beta \sigma}\right\}\right] \\
= & \omega_{0} e^{-\beta t}+\frac{\omega_{0} \varphi_{0} \alpha}{(\beta-\gamma)}\left[e^{-\gamma t}-e^{-\beta t}\right], \\
\varphi= & S^{-1}\left\{\frac{\varphi_{0} \sigma}{\varepsilon+\gamma \sigma}\right\}=\varphi_{0} S^{-1}\left\{\frac{\sigma}{\varepsilon+\gamma \sigma}\right\}=\varphi_{0} e^{-\gamma t} .
\end{aligned}
$$

Equations (17) and (18) give the required number of infected cells $\omega$ and concentration $\varphi$ of viral particles in plasma during HIV-1 infections.

For explaining the importance of equations (17) and (18), we consider different combinations of the values of the factors, namely, the number of potentially infected cells at the beginning of therapy $\omega_{0}$, rate of loss of virus producing cells $\beta$, initial concentration of viral particles in plasma $\varphi_{0}$, rate of infection $\alpha$, and rate constant of viral particle clearance $\gamma$, and determine the corresponding values of $\omega$ and $\varphi$ for different values of time $t$. These values are represented in the Tables 1-7.

From Table 1, it can be concluded that as time $t$ increases from 0 to 1 day, the concentration of viral particles in plasma $\varphi$ decreases for all the five combinations, namely,

$$
\left\{\begin{array}{l}
\varphi_{0}=15 \text { copies } / \mathrm{mL}, \gamma=2.08 \text { day }^{-1} \\
\varphi_{0}=15 \text { copies } / \mathrm{mL}, \gamma=2.09 \text { day }^{-1} \\
\varphi_{0}=15 \text { copies } / \mathrm{mL}, \gamma=2.10 \text { day }^{-1} \\
\varphi_{0}=15 \text { copies } / \mathrm{mL}, \gamma=2.11 \text { day }^{-1} \\
\varphi_{0}=15 \text { copies } / \mathrm{mL}, \gamma=2.12 \text { day }^{-1}
\end{array}\right\} \text { From this table, it is }
$$

also clear that the concentration of viral particles in plasma $\varphi$ decreases as rate constant of viral particle clearance $\gamma$ increases from $2.08 \mathrm{day}^{-1}$ to $2.12 \mathrm{day}^{-1}$. Figure 1 supports the results of Table 1 .

Table 2 shows that as time $t$ increases from 0 to 1 day, the concentration of viral particles in plasma $\varphi$ decreases for all the five combinations, namely, $\left\{\begin{array}{l}\varphi_{0}=15 \text { copies } / \mathrm{mL}, \gamma=2.08 \mathrm{day}^{-1} \\ \varphi_{0}=16 \text { copies } / \mathrm{mL}, \gamma=2.08 \mathrm{day}^{-1} \\ \varphi_{0}=17 \text { copies } / \mathrm{mL}, \gamma=2.08 \mathrm{day}^{-1} \\ \varphi_{0}=18 \text { copies } / \mathrm{mL}, \gamma=2.08 \text { day }^{-1} \\ \varphi_{0}=19 \text { copies } / \mathrm{mL}, \gamma=2.08 \text { day }^{-1}\end{array}\right\}$. From this table, it is also clear that the concentration of viral particles in plasma $\varphi$ increases as initial concentration of viral particles in plasma $\varphi_{0}$ increases from 15 copies $/ \mathrm{mL}$ to 19 copies $/ \mathrm{mL}$. The graph plotted in Figure 2 supports the results of Table 2 .

From Table 3, it can be concluded that as therapy time $t$ increases from 0 to 10 days, the number of infected cells $\omega$ decreases for all the five combinations, namely, $\left\{\begin{array}{l}\omega_{0}=500, \varphi_{0}=15 \text { copies } / \mathrm{mL}, \alpha=0.10 \text { day }^{-1}, \gamma=2.08 \text { day }^{-1}, \beta=0.65 \text { day }^{-1} \\ \omega_{0}=500, \varphi_{0}=15 \text { copies } / \mathrm{mL}, \alpha=0.10 \text { day }^{-1}, \gamma=2.08 \text { day }^{-1}, \beta=0.66 \text { day }^{-1} \\ \omega_{0}=500, \varphi_{0}=15 \text { copies } / \mathrm{mL}, \alpha=0.10 \text { day }^{-1}, \gamma=2.08 \text { day }^{-1}, \beta=0.67 \text { day }^{-1} \\ \omega_{0}=500, \varphi_{0}=15 \text { copies } / \mathrm{mL}, \alpha=0.10 \text { day }^{-1}, \gamma=2.08 \text { day }^{-1}, \beta=0.68 \text { day }^{-1} \\ \omega_{0}=500, \varphi_{0}=15 \text { copies } / \mathrm{mL}, \alpha=0.10 \text { day }^{-1}, \gamma=2.08 \text { day }^{-1}, \beta=0.69 \text { day }^{-1}\end{array}\right\}$.

From this table, it is also clear that the number of infected cells $\omega$ decreases as rate of loss of virus producing cells $\beta$ increases from 0.65 day $^{-1}$ to 0.69 day $^{-1}$. The graph of Figure 3 supports the results of Table 3 .

From Table 4, it can be concluded that as therapy time $t$ increases from 0 to 10 days, the number of infected cells $\omega$ decreases for all the five combinations, namely, $\left\{\begin{array}{l}\omega_{0}=500, \varphi_{0}=15 \text { copies } / \mathrm{mL}, \alpha=0.10 \text { day }^{-1}, \gamma=2.08 \text { day }^{-1}, \beta=0.65 \text { day }^{-1} \\ \omega_{0}=502, \varphi_{0}=15 \text { copies } / \mathrm{mL}, \alpha=0.10 \text { day }^{-1}, \gamma=2.08 \text { day }^{-1}, \beta=0.65 \text { day }^{-1} \\ \omega_{0}=504, \varphi_{0}=15 \text { copies } / \mathrm{mL}, \alpha=0.10 \text { day }^{-1}, \gamma=2.08 \text { day }^{-1}, \beta=0.65 \text { day }^{-1} \\ \omega_{0}=506, \varphi_{0}=15 \text { copies } / \mathrm{mL}, \alpha=0.10 \text { day }^{-1}, \gamma=2.08 \text { day }^{-1}, \beta=0.65 \text { day }^{-1} \\ \omega_{0}=508, \varphi_{0}=15 \text { copies } / \mathrm{mL}, \alpha=0.10 \text { day }^{-1}, \gamma=2.08 \text { day }^{-1}, \beta=0.65 \text { day }^{-1}\end{array}\right\}$.

From this table, it is also clear that the number of infected cells $\omega$ increases as number of potentially infected cells at the beginning of therapy $\omega_{0}$ increases from 500 to 508 . Figure 4 supports the results of Table 4 .

From Table 5, it can be concluded that as therapy time $t$ increases from 0 to 10 days, the number of infected cells $\omega$ decreases for all the five combinations, namely, 
TABLE 1: Concentration of viral particles in plasma $\varphi$ for different values of time $t$ and rate constant of viral particle clearance $\gamma$ with initial concentration of viral particles in plasma $\varphi_{0}=15$ copies $/ \mathrm{mL}$ (see Figure 1).

\begin{tabular}{|c|c|c|c|c|c|}
\hline Time, $t$ (days) & $\gamma=2.08$ day $^{-1}$ & $\gamma=2.09 \mathrm{day}^{-1}$ & $\begin{array}{c}\varphi_{0}=15 \text { copies } / \mathrm{m} \\
\gamma=2.10 \text { day }^{-1}\end{array}$ & $\gamma=2.11 \mathrm{day}^{-1}$ & $\gamma=2.12 \mathrm{day}^{-1}$ \\
\hline 0 & 15 & 15 & 15 & 15 & 15 \\
\hline 0.10 & 12.18 & 12.17 & 12.16 & 12.15 & 12.13 \\
\hline 0.20 & 9.90 & 9.88 & 9.86 & 9.84 & 9.82 \\
\hline 0.30 & 8.04 & 8.01 & 7.99 & 7.96 & 7.94 \\
\hline 0.40 & 6.53 & 6.50 & 6.48 & 6.45 & 6.42 \\
\hline 0.50 & 5.30 & 5.28 & 5.25 & 5.22 & 5.20 \\
\hline 0.60 & 4.31 & 4.28 & 4.25 & 4.23 & 4.20 \\
\hline 0.70 & 3.50 & 3.47 & 3.45 & 3.42 & 3.40 \\
\hline 0.80 & 2.84 & 2.82 & 2.80 & 2.77 & 2.75 \\
\hline 0.90 & 2.31 & 2.29 & 2.27 & 2.25 & 2.23 \\
\hline 1.00 & 1.87 & 1.86 & 1.84 & 1.82 & 1.80 \\
\hline
\end{tabular}

TABLE 2: Concentration of viral particles in plasma $\varphi$ for different values of time $t$ and initial concentration of viral particles in plasma $\varphi_{0}$ with rate constant of viral particle clearance $\gamma=2.08$ day $^{-1}$ (see Figure 2).

\begin{tabular}{|c|c|c|c|c|c|}
\hline \multirow{2}{*}{ Time, $t$ (days) } & \multicolumn{5}{|c|}{$\gamma=2.08 \mathrm{day}^{-1}$} \\
\hline & $\varphi_{0}=15$ copies $/ \mathrm{mL}$ & $\varphi_{0}=16$ copies $/ \mathrm{mL}$ & $\varphi_{0}=17$ copies $/ \mathrm{mL}$ & $\varphi_{0}=18$ copies $/ \mathrm{mL}$ & $\varphi_{0}=19$ copies $/ \mathrm{mL}$ \\
\hline 0 & 15 & 16 & 17 & 18 & 19 \\
\hline 0.10 & 12.18 & 13.00 & 13.81 & 14.62 & 15.43 \\
\hline 0.20 & 9.90 & 10.55 & 11.21 & 11.87 & 12.53 \\
\hline 0.30 & 8.04 & 8.57 & 9.11 & 9.64 & 10.18 \\
\hline 0.40 & 6.53 & 6.96 & 7.40 & 7.83 & 8.27 \\
\hline 0.50 & 5.30 & 5.66 & 6.01 & 6.36 & 6.72 \\
\hline 0.60 & 4.31 & 4.59 & 4.88 & 5.17 & 5.45 \\
\hline 0.70 & 3.50 & 3.73 & 3.96 & 4.20 & 4.43 \\
\hline 0.80 & 2.84 & 3.03 & 3.22 & 3.41 & 3.60 \\
\hline 0.90 & 2.31 & 2.46 & 2.61 & 2.77 & 2.92 \\
\hline 1.00 & 1.87 & 2.00 & 2.12 & 2.25 & 2.37 \\
\hline
\end{tabular}

TABle 3: The number of infected cells $\omega$ for different values of time $t$ and rate of loss of virus producing cells $\beta$ with $\omega_{0}=500$, $\varphi_{0}=$ $15 \mathrm{copies} / \mathrm{mL}, \alpha=0.10 \mathrm{day}^{-1}$, and $\gamma=2.08 \mathrm{day}^{-1}$ (see Figure 3).

\begin{tabular}{|c|c|c|c|c|c|}
\hline \multirow{2}{*}{ Time, $t$ (days) } & \multicolumn{5}{|c|}{$\omega_{0}=500, \varphi_{0}=15$ copies $/ \mathrm{mL}, \alpha=0.10$ day $^{-1}, \gamma=2.08$ day $^{-1}$} \\
\hline & $\beta=0.65$ day $^{-1}$ & $\beta=0.66$ day $^{-1}$ & $\beta=0.67$ day $^{-1}$ & $\beta=0.68$ day $^{-1}$ & $\beta=0.69$ day $^{-1}$ \\
\hline 0 & 500 & 500 & 500 & 500 & 500 \\
\hline 1 & 469.3 & 465.43 & 461.59 & 457.78 & 454.01 \\
\hline 2 & 271.02 & 266.42 & 261.9 & 257.47 & 253.11 \\
\hline 3 & 144.73 & 140.93 & 137.23 & 133.63 & 130.13 \\
\hline 4 & 75.96 & 73.24 & 70.62 & 68.10 & 65.66 \\
\hline 5 & 39.71 & 37.91 & 36.19 & 34.55 & 32.99 \\
\hline 6 & 20.74 & 19.6 & 18.52 & 17.51 & 16.55 \\
\hline 7 & 10.83 & 10.13 & 9.48 & 8.87 & 8.30 \\
\hline 8 & 5.65 & 5.24 & 4.85 & 4.49 & 4.16 \\
\hline 9 & 2.95 & 2.71 & 2.48 & 2.28 & 2.09 \\
\hline 10 & 1.54 & 1.40 & 1.27 & 1.15 & 1.05 \\
\hline
\end{tabular}

$\left\{\begin{array}{l}\omega_{0}=500, \varphi_{0}=15 \text { copies } / \mathrm{mL}, \alpha=0.10 \text { day }^{-1}, \gamma=2.08 \text { day }^{-1}, \beta=0.65 \text { day }^{-1} \\ \omega_{0}=500, \varphi_{0}=16 \text { copies } / \mathrm{mL}, \alpha=0.10 \text { day }^{-1}, \gamma=2.08 \text { day }^{-1}, \beta=0.65 \text { day }^{-1} \\ \omega_{0}=500, \varphi_{0}=17 \text { copies } / \mathrm{mL}, \alpha=0.10 \text { day }^{-1}, \gamma=2.08 \text { day }^{-1}, \beta=0.65 \text { day }^{-1} \\ \omega_{0}=500, \varphi_{0}=18 \text { copies } / \mathrm{mL}, \alpha=0.10 \text { day }^{-1}, \gamma=2.08 \text { day }^{-1}, \beta=0.65 \text { day }^{-1} \\ \omega_{0}=500, \varphi_{0}=19 \text { copies } / \mathrm{mL}, \alpha=0.10 \text { day }^{-1}, \gamma=2.08 \text { day }^{-1}, \beta=0.65 \text { day }^{-1}\end{array}\right\}$.
From this table, it is also clear that the number of infected cells $\omega$ increases as initial concentration of viral particles in plasma $\varphi_{0}$ increases from 15 copies $/ \mathrm{mL}$ to 19 copies $/ \mathrm{mL}$. The graph plotted in Figure 5 supports the results of Table 5. 
TABLE 4: The number of infected cells $\omega$ for different values of time $t$ and number of potentially infected cells at the beginning of therapy $\omega_{0}$ with $\beta=0.65$ day $^{-1}, \varphi_{0}=15$ copies $/ \mathrm{mL}, \alpha=0.10$ day $^{-1}$, and $\gamma=2.08$ day $^{-1}$ (see Figure 4 ).

\begin{tabular}{|c|c|c|c|c|c|}
\hline \multirow{2}{*}{ Time, $t$ (days) } & \multicolumn{5}{|c|}{$\beta=0.65$ day $^{-1}, \varphi_{0}=15$ copies $/ \mathrm{mL}, \alpha=0.10$ day $^{-1}, \gamma=2.08$ day $^{-1}$} \\
\hline & $\omega_{0}=500$ & $\omega_{0}=502$ & $\omega_{0}=504$ & $\omega_{0}=506$ & $\omega_{0}=508$ \\
\hline 0 & 500 & 502 & 504 & 506 & 508 \\
\hline 1 & 469.30 & 471.18 & 473.05 & 474.93 & 476.81 \\
\hline 2 & 271.02 & 272.10 & 273.18 & 274.27 & 275.35 \\
\hline 3 & 144.73 & 145.31 & 145.89 & 146.47 & 147.05 \\
\hline 4 & 75.96 & 76.27 & 76.57 & 76.88 & 77.18 \\
\hline 5 & 39.71 & 39.87 & 40.02 & 40.18 & 40.34 \\
\hline 6 & 20.74 & 20.82 & 20.9 & 20.98 & 21.07 \\
\hline 7 & 10.83 & 10.87 & 10.91 & 10.96 & 11.00 \\
\hline 8 & 5.65 & 5.67 & 5.70 & 5.72 & 5.74 \\
\hline 9 & 2.95 & 2.96 & 2.97 & 2.99 & 3.00 \\
\hline 10 & 1.54 & 1.55 & 1.55 & 1.56 & 1.56 \\
\hline
\end{tabular}

TABLE 5: The number of infected cells $\omega$ for different values of time $t$ and initial concentration of viral particles in plasma $\varphi_{0}$ with $\beta=0.65$ day $^{-1}, \omega_{0}=500, \alpha=0.10$ day $^{-1}$, and $\gamma=2.08$ day $^{-1}$ (see Figure 5).

\begin{tabular}{|c|c|c|c|c|c|}
\hline \multirow{2}{*}{ Time, $t$ (days) } & \multicolumn{5}{|c|}{$\beta=0.65$ day $^{-1}, \omega_{0}=500, \alpha=0.10$ day $^{-1}, \gamma=2.08$ day $^{-1}$} \\
\hline & $\varphi_{0}=15$ copies $/ \mathrm{mL}$ & $\varphi_{0}=16$ copies $/ \mathrm{mL}$ & $\varphi_{0}=17$ copies $/ \mathrm{mL}$ & $\varphi_{0}=18$ copies $/ \mathrm{mL}$ & $\varphi_{0}=19$ copies $/ \mathrm{mL}$ \\
\hline 0 & 500 & 500 & 500 & 500 & 500 \\
\hline 1 & 469.3 & 483.19 & 497.07 & 510.96 & 524.84 \\
\hline 2 & 271.02 & 280.00 & 288.98 & 297.97 & 306.95 \\
\hline 3 & 144.73 & 149.64 & 154.55 & 159.45 & 164.36 \\
\hline 4 & 75.96 & 78.55 & 81.14 & 83.73 & 86.32 \\
\hline 5 & 39.71 & 41.06 & 42.42 & 43.77 & 45.13 \\
\hline 6 & 20.74 & 21.44 & 22.15 & 22.86 & 23.57 \\
\hline 7 & 10.83 & 11.20 & 11.56 & 11.93 & 12.30 \\
\hline 8 & 5.65 & 5.84 & 6.04 & 6.23 & 6.42 \\
\hline 9 & 2.95 & 3.05 & 3.15 & 3.25 & 3.35 \\
\hline 10 & 1.54 & 1.59 & 1.65 & 1.70 & 1.75 \\
\hline
\end{tabular}

TABLE 6: The number of infected cells $\omega$ for different values of time $t$ and rate of infection $\alpha$ with $\varphi_{0}=15$ copies $/ \mathrm{mL}, \beta=0.65$ day ${ }^{-1}, \omega_{0}=$ 500, and $\gamma=2.08 \mathrm{day}^{-1}$ (see Figure 6).

\begin{tabular}{|c|c|c|c|c|c|}
\hline \multirow{2}{*}{ Time, $t$ (days) } & \multicolumn{5}{|c|}{$\varphi_{0}=15$ copies $/ \mathrm{mL}, \beta=0.65 \mathrm{day}^{-1}, \omega_{0}=500, \gamma=2.08 \mathrm{day}^{-1}$} \\
\hline & $\alpha=0.06$ day $^{-1}$ & $\alpha=0.07 \mathrm{day}^{-1}$ & $\alpha=0.08$ day $^{-1}$ & $\alpha=0.09$ day $^{-1}$ & $\alpha=0.10$ day $^{-1}$ \\
\hline 0 & 500 & 500 & 500 & 500 & 500 \\
\hline 1 & 385.99 & 406.82 & 427.64 & 448.47 & 469.30 \\
\hline 2 & 217.12 & 230.59 & 244.07 & 257.54 & 271.02 \\
\hline 3 & 115.30 & 122.65 & 130.01 & 137.37 & 144.73 \\
\hline 4 & 60.43 & 64.32 & 68.20 & 72.08 & 75.96 \\
\hline 5 & 31.58 & 33.61 & 35.64 & 37.68 & 39.71 \\
\hline 6 & 16.49 & 17.55 & 18.61 & 19.67 & 20.74 \\
\hline 7 & 8.61 & 9.16 & 9.72 & 10.27 & 10.83 \\
\hline 8 & 4.49 & 4.78 & 5.07 & 5.36 & 5.65 \\
\hline 9 & 2.35 & 2.50 & 2.65 & 2.80 & 2.95 \\
\hline 10 & 1.22 & 1.30 & 1.38 & 1.46 & 1.54 \\
\hline
\end{tabular}

Table 6 shows that as therapy time $t$ increases from 0 to 10 days, the number of infected cells $\omega$ decreases for all the five combinations,

\footnotetext{
$\omega_{0}=500, \varphi_{0}=15$ copies $\left./ \mathrm{mL}, \alpha=0.06 \mathrm{day}^{-1}, \gamma=2.08 \mathrm{day}^{-1}, \beta=0.65 \mathrm{day}^{-1}\right)$

$\left.\begin{array}{l}\omega_{0}=500, \varphi_{0}=15 \text { copies } / \mathrm{mL}, \alpha=0.06 \mathrm{day}^{-1}, \gamma=2.08 \mathrm{day}^{-1}, \beta=0.65 \mathrm{day}^{-1} \\ \omega_{0}=500, \varphi_{0}=15 \text { copies } / \mathrm{mL}, \alpha=0.07 \text { day }^{-1}, \gamma=2.08 \text { day }^{-1}, \beta=0.65 \text { day }^{-1} \\ \omega_{0}=500, \varphi_{0}=15 \text { copes }\end{array}\right\}$

$\omega_{0}=500, \varphi_{0}=15$ copies $/ \mathrm{mL}, \alpha=0.08$ day $^{-1}, \gamma=2.08$ day $^{-1}, \beta=0.65$ day $\left.^{-1}\right\}$.

$\omega_{0}=500, \varphi_{0}=15$ copies $/ \mathrm{mL}, \alpha=0.09$ day $^{-1}, \gamma=2.08$ day $^{-1}, \beta=0.65$ day $^{-1}$

$\omega_{0}=500, \varphi_{0}=15$ copies $/ \mathrm{mL}, \alpha=0.10$ day $^{-1}, \gamma=2.08$ day $^{-1}, \beta=0.65$ day $^{-1}$
}

From this table, it is also clear that the number of infected cells $\omega$ increases as rate of infection $\alpha$ increases from $0.06 \mathrm{day}^{-1}$ to $0.10 \mathrm{day}^{-1}$. From the graph plotted in Figure 6, it is also clear that the number of infected cells $\omega$ increases as rate of infection $\alpha$ increases from 0.06 day $^{-1}$ to 0.10 day $^{-1}$.

Table 7 shows that as therapy time $t$ increases from 0 to 10 days, the number of infected cells $\omega$ decreases for all the 
TABLE 7: The number of infected cells $\omega$ for different values of time $t$ and rate constant of viral particle clearance $\gamma$ with $\varphi_{0}=15$ copies $/ \mathrm{mL}, \beta=0.65$ day $^{-1}, \omega_{0}=500$, and $\gamma=2.08$ day $^{-1}$ (see Figure 7).

\begin{tabular}{|c|c|c|c|c|c|}
\hline \multirow{2}{*}{ Time, $t$ (days) } & \multicolumn{5}{|c|}{$\varphi_{0}=15$ copies $/ \mathrm{mL}, \beta=0.65$ day $^{-1}, \omega_{0}=500, \gamma=2.08$ day $^{-1}$} \\
\hline & $\gamma=2.08 \mathrm{day}^{-1}$ & $\gamma=2.09 \mathrm{day}^{-1}$ & $\gamma=2.10 \mathrm{day}^{-1}$ & $\gamma=2.11 \mathrm{day}^{-1}$ & $\gamma=2.12 \mathrm{day}^{-1}$ \\
\hline 0 & 500 & 500 & 500 & 500 & 500 \\
\hline 1 & 469.30 & 468.50 & 467.71 & 466.92 & 466.13 \\
\hline 2 & 271.02 & 270.24 & 269.47 & 268.71 & 267.96 \\
\hline 3 & 144.73 & 144.25 & 143.78 & 143.31 & 142.84 \\
\hline 4 & 75.96 & 75.70 & 75.44 & 75.18 & 74.93 \\
\hline 5 & 39.71 & 39.57 & 39.43 & 39.29 & 39.16 \\
\hline 6 & 20.74 & 20.66 & 20.59 & 20.52 & 20.45 \\
\hline 7 & 10.83 & 10.79 & 10.75 & 10.71 & 10.67 \\
\hline 8 & 5.65 & 5.63 & 5.61 & 5.59 & 5.57 \\
\hline 9 & 2.95 & 2.94 & 2.93 & 2.92 & 2.91 \\
\hline 10 & 1.54 & 1.53 & 1.53 & 1.52 & 1.52 \\
\hline
\end{tabular}
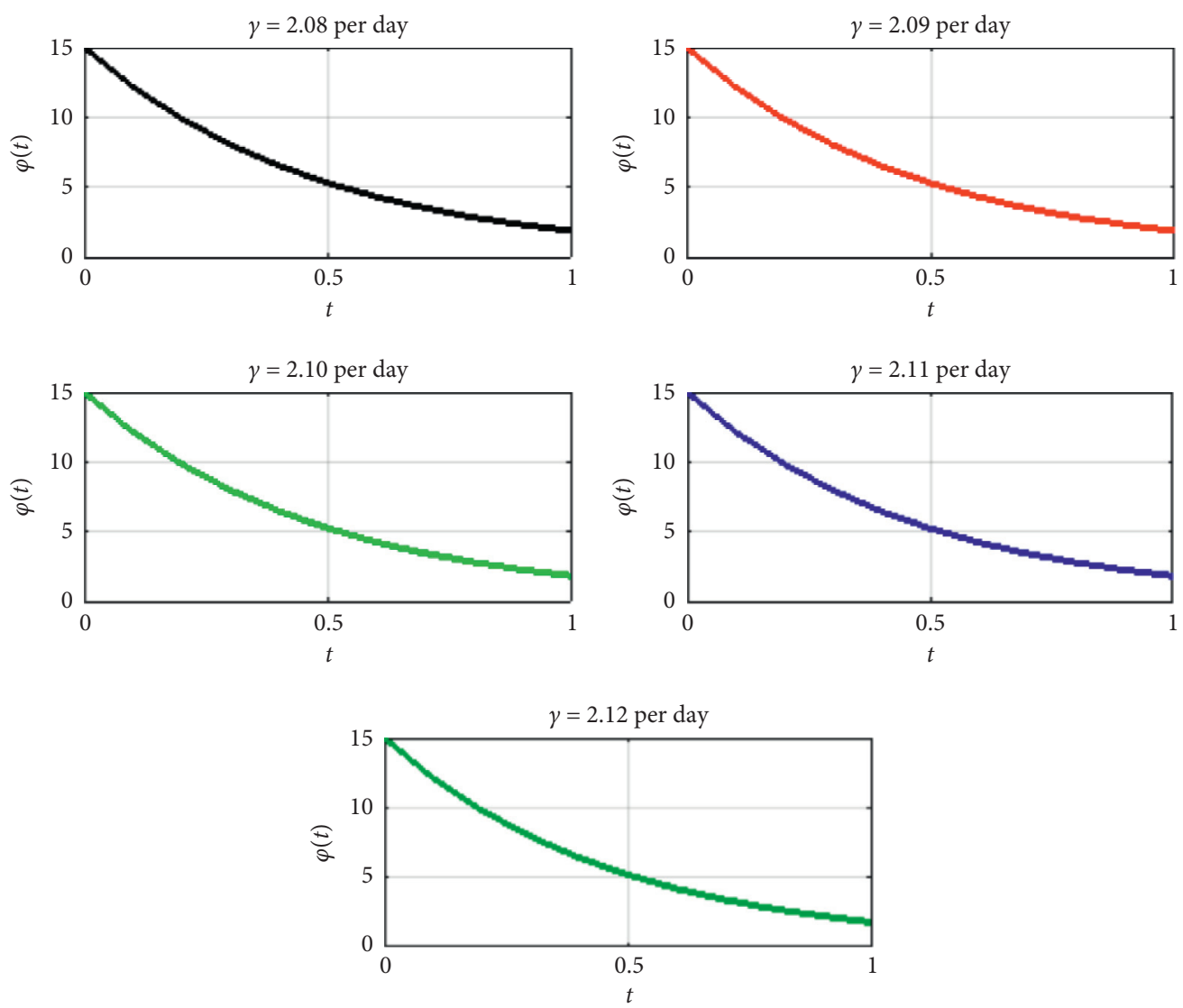

FIGURE 1: Concentration of viral particles in plasma $\varphi$ for different values of time $t$ and rate constant of viral particle clearance $\gamma$ with initial concentration of viral particles in plasma $\varphi_{0}=15$ copies $/ \mathrm{mL}$.

five combinations, namely, $\left\{\begin{array}{l}\omega_{0}=500, \varphi_{0}=15 \text { copies } / \mathrm{mL}, \alpha=0.10 \text { day }^{-1}, \gamma=2.08 \text { day }^{-1}, \beta=0.65 \text { day }^{-1} \\ \omega_{0}=500, \varphi_{0}=15 \text { copies } / \mathrm{mL}, \alpha=0.10 \text { day }^{-1}, \gamma=2.09 \text { day }^{-1}, \beta=0.65 \text { day }^{-1} \\ \omega_{0}=500, \varphi_{0}=15 \text { copies } / \mathrm{mL}, \alpha=0.10 \text { day }^{-1}, \gamma=2.10 \text { day }^{-1}, \beta=0.65 \text { day }^{-1} \\ \omega_{0}=500, \varphi_{0}=15 \text { copies } / \mathrm{mL}, \alpha=0.10 \text { day }^{-1}, \gamma=2.11 \text { day }^{-1}, \beta=0.65 \text { day }^{-1} \\ \omega_{0}=500, \varphi_{0}=15 \text { copies } / \mathrm{mL}, \alpha=0.10 \text { day }^{-1}, \gamma=2.12 \text { day }^{-1}, \beta=0.65 \text { day }^{-1}\end{array}\right\}$.
From this table, it is also clear that the number of infected cells $\omega$ decreases as rate constant of viral particle clearance $\gamma$ increases from $2.08 \mathrm{day}^{-1}$ to $2.12 \mathrm{day}^{-1}$. Figure 7 supports the results of Table 7 . 


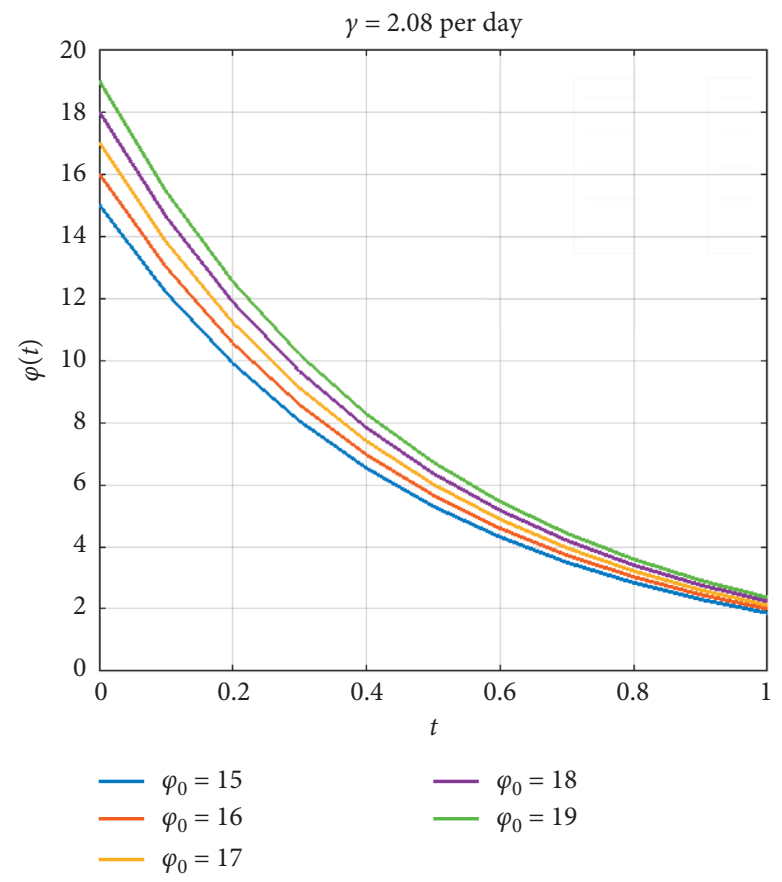

FIGURE 2: Concentration of viral particles in plasma $\varphi$ for different values of time $t$ and initial concentration of viral particles in plasma $\varphi_{0}$ with rate constant of viral particle clearance $\gamma=2.08$ day $^{-1}$.

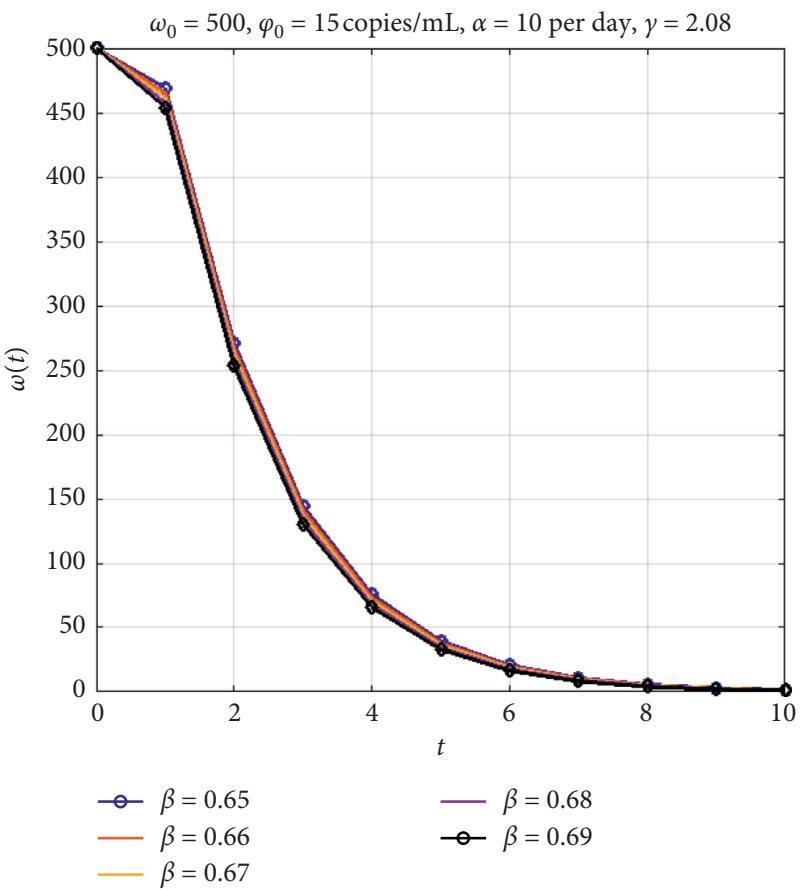

Figure 3: The number of infected cells $\omega$ for different values of time $t$ and rate of loss of virus producing cells $\beta$ with $\omega_{0}=500, \varphi_{0}=15$ copies $/ \mathrm{mL}, \alpha=0.10$ day $^{-1}$, and $\gamma=2.08$ day $^{-1}$. 


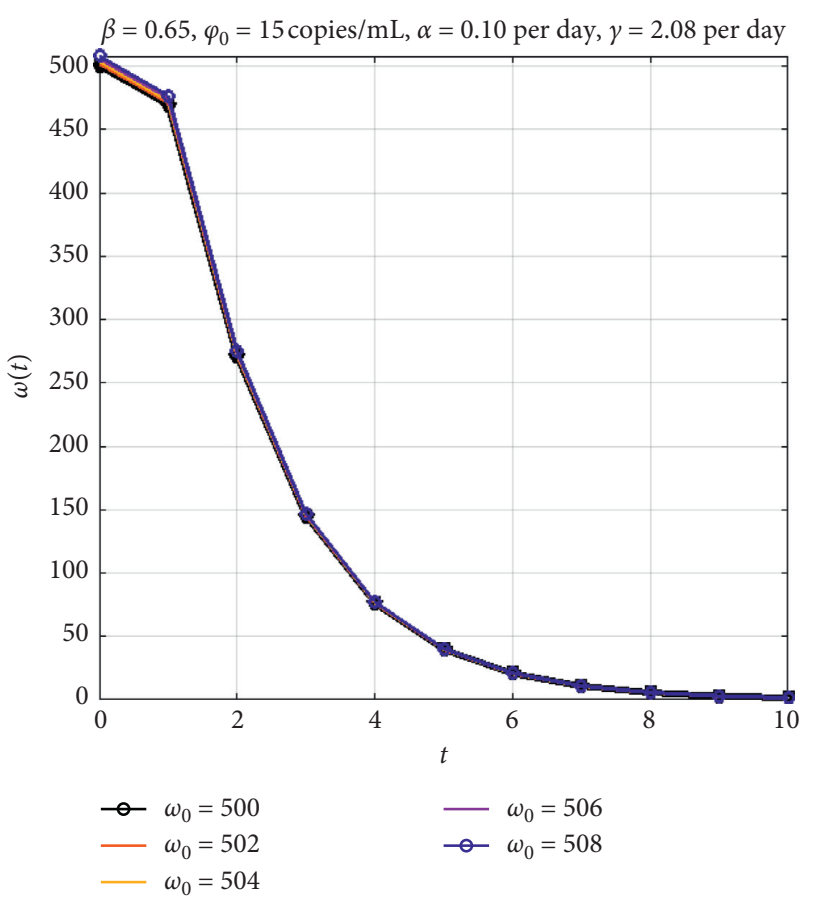

FIGURE 4: The number of infected cells $\omega$ for different values of time $t$ and number of potentially infected cells at the beginning of therapy $\omega_{0}$ with $\beta=0.65$ day $^{-1}, \varphi_{0}=15$ copies $/ \mathrm{mL}, \alpha=0.10$ day $^{-1}$, and $\gamma=2.08$ day $^{-1}$.

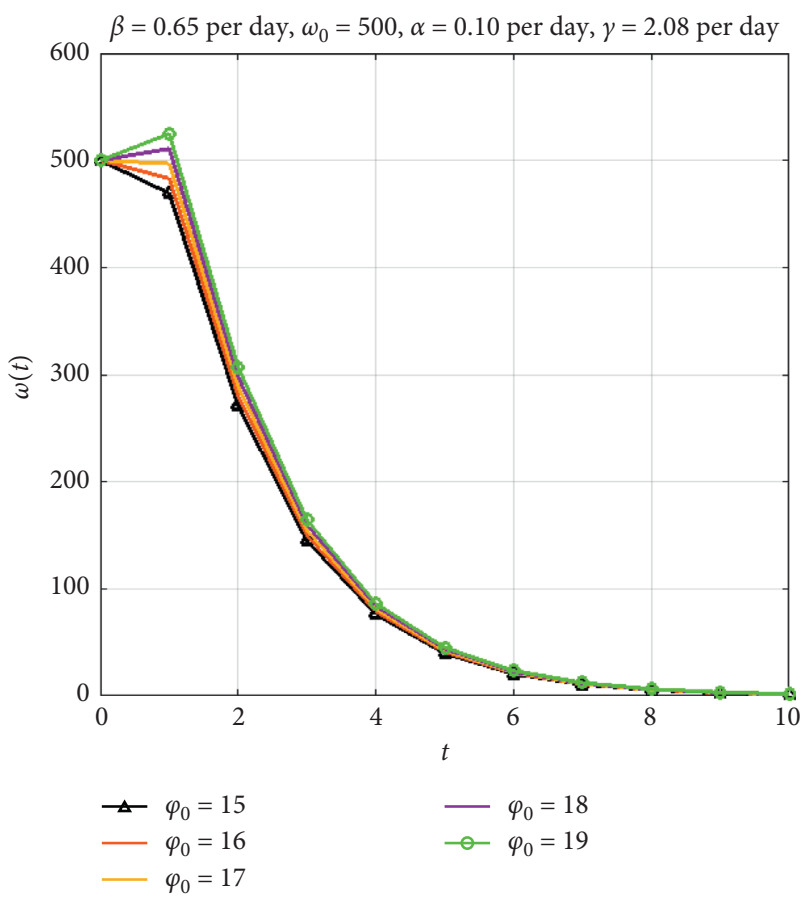

Figure 5: The number of infected cells $\omega$ for different values of time $t$ and initial concentration of viral particles in plasma $\varphi_{0}$ with $\beta=0.65$ day $^{-1}, \omega_{0}=500, \alpha=0.10$ day $^{-1}$, and $\gamma=2.08$ day $^{-1}$. 


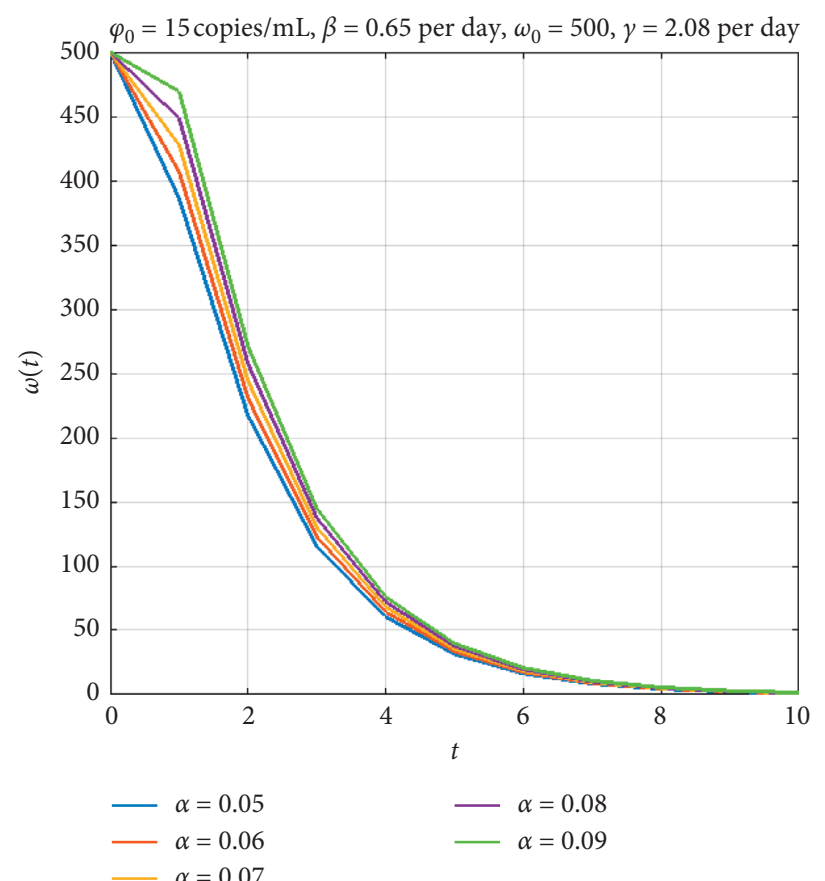

Figure 6: The number of infected cells $\omega$ for different values of time $t$ and rate of infection $\alpha$ with $\varphi_{0}=15$ copies $/ \mathrm{mL}, \beta=0.65$ day $^{-1}, \omega_{0}=500$, and $\gamma=2.08$ day $^{-1}$.

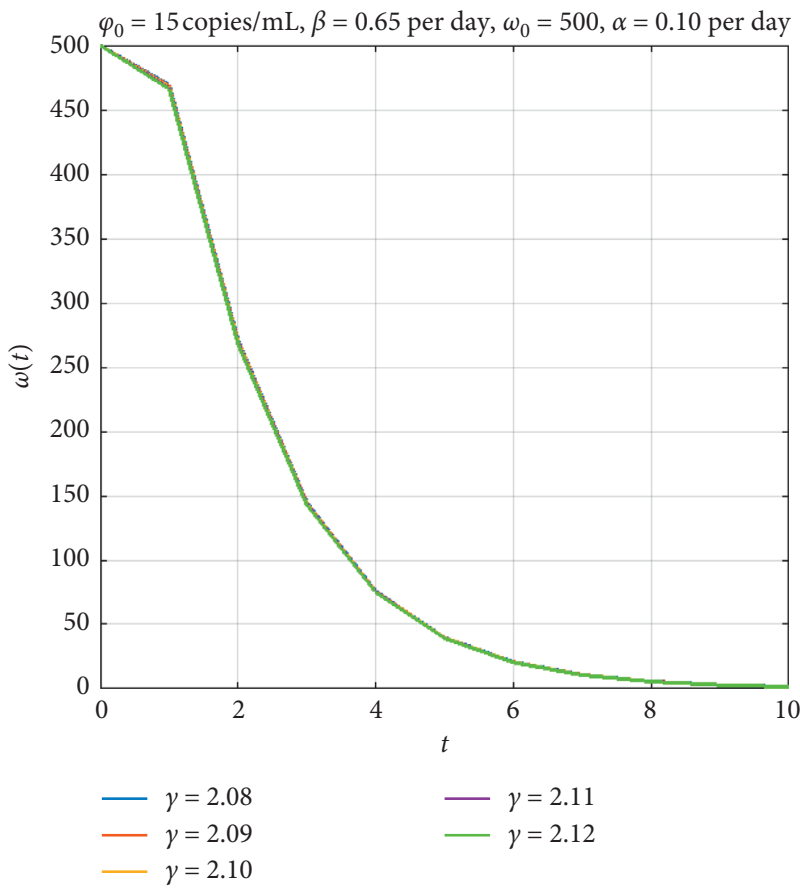

Figure 7: The number of infected cells $\omega$ for different values of time $t$ and rate constant of viral particle clearance $\gamma$ with $\varphi_{0}=15$ copies $/ \mathrm{mL}, \beta=0.65$ day $^{-1}, \omega_{0}=500$, and $\alpha=0.10$ day $^{-1}$. 
TABLE 8: Shehu transformation of frequently used functions [12-15].

\begin{tabular}{lcc}
\hline S.N. & $\omega(t)$ & $S\{\omega(t)\}=T(\varepsilon, \sigma)$ \\
\hline 1 & 1 & $(\sigma / \varepsilon)$ \\
2 & $t$ & $(\sigma / \varepsilon)^{2}$ \\
3 & $t^{2}$ & $2(\sigma / \varepsilon)^{3}$ \\
4 & $t^{\rho}, \rho \in N$ & $\rho !(\sigma / \varepsilon)^{\rho+1}$ \\
5 & $t^{\rho}, \rho>-1$ & $\Gamma(\rho+1)(\sigma / \varepsilon)^{\rho+1}$ \\
6 & $e^{\text {lt }}$ & $(\sigma / \varepsilon-l \sigma)$ \\
7 & $\operatorname{sinlt}$ & $\left(l \sigma^{2} /\left(\varepsilon^{2}+l^{2} \sigma^{2}\right)\right)$ \\
8 & $\cos l t$ & $\left(\sigma \varepsilon /\left(\varepsilon^{2}+l^{2} \sigma^{2}\right)\right)$ \\
9 & $\operatorname{sinhlt}$ & $\left(l \sigma^{2} /\left(\varepsilon^{2}-l^{2} \sigma^{2}\right)\right)$ \\
10 & $\operatorname{coshlt}$ & $\left(\sigma \varepsilon /\left(\varepsilon^{2}-l^{2} \sigma^{2}\right)\right)$ \\
11 & $J_{0}(l \mathrm{lt})$ & $\left(\sigma / \sqrt{\left(\varepsilon^{2}+l^{2} \sigma^{2}\right)}\right)$ \\
\hline
\end{tabular}

TABLE 9: Inverse Shehu transformation of frequently used functions [12-14].

\begin{tabular}{lcc}
\hline S.N. & $T(\varepsilon, \sigma)$ & $\omega(t)=S^{-1}\{T(\varepsilon, \sigma)\}$ \\
\hline 1 & $(\sigma / \varepsilon)$ & 1 \\
2 & $(\sigma / \varepsilon)^{2}$ & $t$ \\
3 & $(\sigma / \varepsilon)^{3}$ & $\left(t^{2} / 2 !\right)$ \\
4 & $(\sigma / \varepsilon)^{\rho+1}, \rho \in N$ & $\left(t^{\rho} / \rho !\right)$ \\
5 & $(\sigma / \varepsilon)^{\rho+1}, \rho>-1$ & $\left(t^{\rho} / \Gamma(\rho+1)\right)$ \\
6 & $(\sigma / \varepsilon-l \sigma)$ & $e^{\mathrm{lt}}$ \\
7 & $\left(\sigma^{2} /\left(\varepsilon^{2}+l^{2} \sigma^{2}\right)\right)$ & $(\operatorname{sinlt} / \mathrm{l})$ \\
8 & $\left(\sigma \varepsilon /\left(\varepsilon^{2}+l^{2} \sigma^{2}\right)\right)$ & $\cos \mathrm{lt}$ \\
9 & $\left(\sigma^{2} /\left(\varepsilon^{2}-l^{2} \sigma^{2}\right)\right)$ & $(\sinh \mathrm{lt} / l)$ \\
10 & $\left(\sigma \varepsilon /\left(\varepsilon^{2}-l^{2} \sigma^{2}\right)\right)$ & $\operatorname{coshlt}$ \\
11 & $\left(\sigma / \sqrt{\left(\varepsilon^{2}+l^{2} \sigma^{2}\right)}\right)$ & $J_{0}(\mathrm{lt})$ \\
\hline
\end{tabular}

Shehu and inverse Shehu transformations of frequently used functions are presented in Tables 8 and 9, respectively.

\section{Conclusions}

In the present paper, the authors fruitfully determined the total number of infected cells and concentration of viral particles in plasma during HIV-1 infections using Shehu transformation. Results depict that Shehu transformation is very effective integral transformation for determining the total number of infected cells and concentration of viral particles in plasma during HIV-1 infections. The proposed method is better than the other methods because it provides the exact solution of critical problems in a very short time and without doing large calculation work. This study is very helpful in medical field and provides the solution of critical problems of determining total number of infected cells and concentration of viral particles in plasma during HIV-1 infections at the time of operation. The solutions of complex problems of science, medicine, space, pharmacokinetics, economics, business, and engineering such as the problem of residential segregation, arms race models, the problem related to drug distribution in the blood, problems of heat and mass transfer, radioactive decay problem, and the problem of tumor growth can be determined by applying Shehu transformation on them in future.

\section{Data Availability}

The datasets used to support the findings of this paper are available from the corresponding author upon request.

\section{Conflicts of Interest}

The authors declare that they have no conflicts of interest.

\section{Acknowledgments}

This research was supported by Taif University Researchers Supporting Project (TURSP-2020/155), Taif University, Taif, Saudi Arabia.

\section{References}

[1] P. M. Sharp, G. M. Shaw, and B. H. Hahn, "Simian immunodeficiency virus infection of chimpanzees," Journal of $\mathrm{Vi}$ rology, vol. 79, no. 7, pp. 3891-3902, 2005.

[2] P. Lemey, O. G. Pybus, B. Wang, N. K. Saksena, M. Salemi, and A.-M. Vandamme, "Tracing the origin and history of the HIV-2 epidemic," Proceedings of the National Academy of Sciences, vol. 100, no. 11, pp. 6588-6592, 2003.

[3] B. H. Hahn, G. M. Shaw, K. M. De Cock, and P. M. Sharp, "AIDS as a zoonosis: scientific and public health implications," Science, vol. 287, no. 5453, pp. 607-614, 2000.

[4] L. Debnath and D. Bhatta, Integral Transforms and Their Applications, Chapman \& Hall/CRC, Boca Raton, FL, USA, 2nd edition, 2007.

[5] L. C. Andrews and B. K. Shivamoggi, Integral Transforms for Engineers, SPIE, Bellingham, WC, USA, 1999.

[6] S. Aggarwal, N. Asthana, and D. P. Singh, "Solution of population growth and decay problems by using aboodh transform method," International Journal of Research in Advent Technology, vol. 6, no. 10, pp. 2706-2710, 2018.

[7] S. Aggarwal, D. P. Singh, N. Asthana, and A. R. Gupta, "Application of Elzaki transform for solving population growth and decay problems," Journal of Emerging Technologies and Innovative Research, vol. 5, no. 9, pp. 281-284, 2018.

[8] S. Aggarwal and R. Chaudhary, "A comparative study of Mohand and Laplace transforms," Journal of Emerging Technologies and Innovative Research, vol. 6, no. 2, pp. 230240, 2019.

[9] S. Aggarwal, N. Sharma, R. Chaudhary, and A. R. Gupta, "A comparative study of ohand and Kamal transforms," Global Journal of Engineering Science and Researches, vol. 6, no. 2, pp. 113-123, 2019.

[10] S. Aggarwal, R. Mishra, and A. Kumar, "A comparative study of Mohand and Elzaki transforms," Global Journal of Engineering Science and Researches, vol. 6, no. 2, pp. 203-213, 2019.

[11] S. Maitama and W. Zhao, "New integral transform: Shehu transform a generalization of Sumudu and Laplace transform 
for solving differential equations," International Journal of Analysis and Applications, vol. 17, no. 2, pp. 167-190, 2019.

[12] S. Aggarwal, S. D. Sharma, and A. R. Gupta, "Application of Shehu transform for handling growth and decay problems," Global Journal of Engineering Science and Researches, vol. 6, no. 4, pp. 190-198, 2019.

[13] S. Aggarwal and A. R. Gupta, "Shehu transform for solving Abel's integral equation," Journal of Emerging Technologies and Innovative Research, vol. 6, no. 5, pp. 101-110, 2019.

[14] S. Aggarwal, A. R. Gupta, and S. D. Sharma, "A new application of Shehu transform for handling volterra integral equations of first kind," International Journal of Research in Advent Technology, vol. 7, no. 4, pp. 439-445, 2019.

[15] S. Aggarwal and G. Pratap Singh, "Shehu transform of error function," International Journal of Research in Advent Technology, vol. 7, no. 6, pp. 54-60, 2019.

[16] H. J. Ricardo, A Modern Introduction to Differential Equations, Academic Press/Elsevier, Amsterdam, Netherlands, 2nd edition, 2009.

[17] C. H. Taubes, Modelling Differential Equations in Biology, Cambridge University Press, Cambridge, UK, 2009.

[18] A. S. Perelson, A. U. Neumann, M. Markowitz, J. M. Leonard, and D. D. Ho, "HIV-1 dynamics in vivo: virion clearance rate, infected cell life-span, and viral generation time," Science, vol. 271, no. 5255, pp. 1582-1586, 1996.

[19] A. S. Perelson and P. W. Nelson, "Mathematical analysis of HIV-1 dynamics in vivo," SIAM Review, vol. 41, no. 1 , pp. 3-44, 1999.

[20] A. S. Perelson, "Modelling viral and immune system dynamics," Nature Reviews Immunology, vol. 2, no. 1, pp. 28-36, 2002.

[21] P. W. Nelson and A. S. Perelson, "Mathematical analysis of delay differential equation models of HIV-1 infection," Mathematical Biosciences, vol. 179, no. 1, pp. 73-94, 2002.

[22] M. A. Stafford, L. Corey, Y. Cao, E. S. Daar, D. D. Ho, and A. S. Perelson, "Modeling plasma virus concentration during primary HIV infection," Journal of Theoretical Biology, vol. 203, no. 3, pp. 285-301, 2000.

[23] L. Rong, Z. Feng, and A. S. Perelson, "Mathematical modeling of HIV-1 infection and drug therapy," Mathematical Modelling of Biosystems, vol. 102, pp. 87-131, 2008.

[24] S. Aggarwal, A. R. Gupta, D. P. Singh, N. Asthana, and N. Kumar, "Application of Laplace transform for solving population growth and decay problems," International Journal of Latest Technology in Engineering, Management \& Applied Science, vol. 7, no. 9, pp. 141-145, 2018. 